Volume 9

Issue 1

2012
$\mathrm{B}$ rain injury, occurring when physical trauma causes brain damage, either closed-head or penetrating, contributes to one third of all injury-related deaths annually and places approximately 275,000 people in the hospital each year (Faul, $\mathrm{Xu}$, Wald, Coronado, 2010; Center for Disease Control and Prevention, 2012). Approximately 1.7 million people in the US sustain a traumatic brain injury (TBI) yearly (Faul, et al, 2010). It is estimated that TBI related medical costs, both direct and indirect, totaled $\$ 76.5$ billion dollars in 2010 (Center for Disease Control and Prevention, 2012).

Amongst the veteran population, TBI is often associated with psychological difficulty and increased risk of substance abuse (Department of Veterans Affairs). More than 203,019 individuals in the US forces had sustained TBI from combat and non-combat related causes (Defense and Veterans Brain Injury Center, 2012). This is approximately a $26 \%$ increase in medically diagnosed cases of US soldiers since 2000 (Rand, 2008). Many of these soldiers suffer from deficits in cognitive and motor functions, as well as emotional difficulties, plaguing these persons with tremendous hardships and frustrations (Forrest, Schwam, $\&$ Cohen, 2002). These deficits commonly leave patients unable to hold employment or function properly in social surroundings; in turn, incurring serious social and economic costs to both the patients and relatives.

More troubling, individuals with TBI may receive improper or no treatment, despite self-reports of persisting deficits post-injury (Huebner, Johnson, Miller Bennett, \& Schneck, 2003). While some communitybased rehabilitative supports exist (e.g. vocational rehabilitation services, or case management), these programs are not widely available for persons with TBI in

\section{Brain Injury Resources}

olleen E. McKay, MA, CAGS \& Meghan E. Heffernan, BA

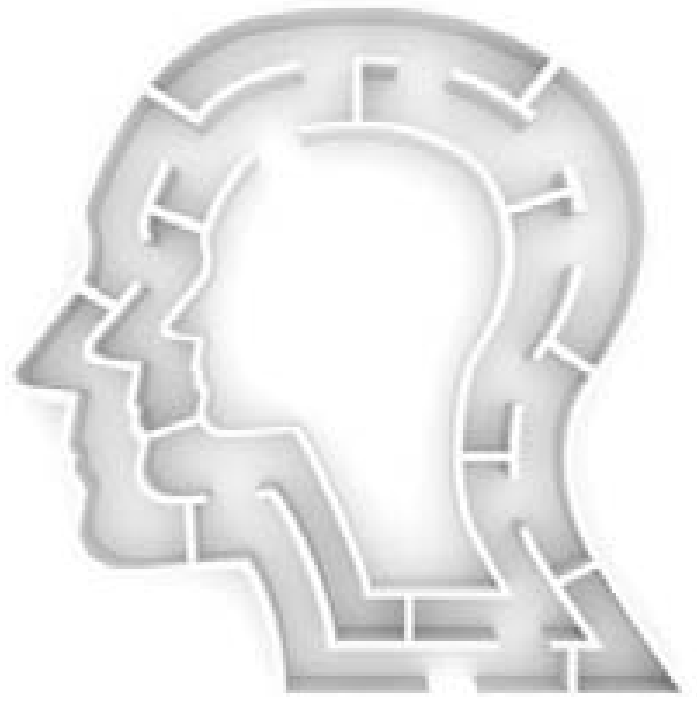

all communities. In all, there is an inadequate service array to support individuals with brain injuries.

\section{Efforts of the University of Massachusetts Medical School (UMMS)}

The Brain Injury Academic Interest Group (BIAIG) in the UMMS Psychiatry Department aims to engage faculty and staff from the department and Commonwealth Medicine, researchers and providers at local and state agencies, and other academic institutions around multiple issues relevant to brain injuries. The BI-AIG explores ongoing work and interests within the BI-AIG to generate projects and priorities for the future.

The BI-AIG discusses issues related to TBI from clinical and research perspectives. BI-AIG goals include developing protocols for TBI follow-up that will standardize assessments and will lay the groundwork for research. The BI-AIG is developing a regional resource list that providers, researchers and individuals with brain injuries can use to access supports in Massachusetts. Below are some resources identified by the BIAIG that provide information about brain injuries. 


\section{Resources for Information about Brain}

Injury

- The Brain Injury Association of American (www. biausa.org) is a nationwide network advocating for TBI awareness and quality health care.

- Brain Line (www.brainline.org) provides information on preventing, treating, and living with traumatic brain injury (TBI).

- The International Brain Injury Association (IBIA) (www.internationalbrain.org) works with professionals, advocates, policy-makers, to improve TBI-related outcomes.

- The Brain Injury Recovery Network (www. tbirecovery.org) provides practical information for brain injury survivors and families.

- The International Brain Injury Clubhouse Association (www.braininjuryclubhouses.net) is a collaborative network of programs that serve persons with brain injury and stroke.

- The Defense and Veterans Brain Injury Center (DVBIC) (www.dvbic.org) serves active duty military, and veterans with TBI through clinical care, innovative research, and educational programs. The DVBIC website has information and resources (e.g. TBI clinical tools).

\section{Resources for Researchers}

- Center on Outcome Measurement in Brain Injury (COMBI) (www.tbims.org/combi) provides information on outcome measures and scales for rehabilitation and assessment of brain injury.

- The TBI Model Systems Knowledge Translation Center (www.msktc.org/tbi/ ) provides information for consumers, a database of research articles, reviews of TBI research, and current research activities.

- The Traumatic Brain Injury Model Systems National Data \& Statistical Center (TBINDSC) (www.tbindsc.org/) provides technical assistance, training, and methodological consultation to the TBIMS centers.

- The National Institute of Neurological Disorders and Stroke (NINDS) (www.ninds.nih.gov/disorders/ tbi/tbi.htm) conducts and funds research on the causes, prevention, diagnosis and treatment of neurological disorders and stroke.

\section{Resources in Massachusetts}

- The Acquired Brain Injury (ABI) \& Traumatic Brain Injury Commission (www.mass.gov/ orgs/braininjurycommission) was established to investigate the rehabilitative residential and integrated community-based support services for persons with acquired brain injury.

- The UMMS ABI Waiver is a collaboration of UMMS, MassHealth, and the Massachusetts Rehabilitation Commission. The Waiver team conducts participant outreach, clinical eligibility screenings, transition, home and community-based services and case management (https://www.mass.gov/info-details/ acquired-brain-injury-abi-waivers)

- The Brain Injury Association of Massachusetts (www.biama.org) is a not-for-profit organization that supports brain injury survivors and their families, that offers educational information, advocacy, and programs to prevent brain injuries.

- The Statewide Head Injury Program (SHIP) (https://www.mass.gov/service-details/statewidehead-injury-program-ship) maintains a network of community-based services and supports that assist individuals with head injuries in maintaining or increasing their level of independence at home, work and in their communities.

\section{Additional Information For Family Members \& Persons Suffering From TBI}

Many family members and persons suffering from TBI do not receive help in how to cope with TBIrelated deficits. We recommend that persons with TBI and their caregivers seek treatment from trained TBI professionals in order to learn how to live with the brain injury, and the resources listed here provide useful information which may help to inform those choices. It is critical to note that only a medically licensed professional can diagnose a person with TBI.

For TBI Service Providers \& Researchers The continued efforts of the BI-AIG include developing resources and stimulating collaborations between researchers and providers. We offer a standing invitation for providers and researchers interested in TBI to attend a BI-AIG session and become a member. For further information regarding the BI-AIG, please contact the authors of this issue brief listed below:

Colleen.McKay@umassmed.edu Meghan.Heffernan@umassmed.edu 


\section{References}

Center for Disease Control and Prevention. (2012). Severe Traumatic Brain Injury. Retrieved February 22, 2012 from http:// www.cdc.gov/traumaticbraininjury/severe.html

Defense and Veterans Brain Injury Center. (2011). DoD Worldwide Numbers for TBI (non-combat and combat injuries). Retrieved February 22, 2012 from http://www.dvbic.org/TBI-Numbers.aspx

Degeneffe, C.E. (2001). Family caregiving and traumatic brain injury. Health and Social Work, 26, 257-268.

Faul, M., Xu, L., Wald, M.M, \& Coronado, V.G. (2010). Traumatic brain injury in the United States: Emergency department visits, hospitalizations, and deaths. Atlanta (GA): Centers for Disease Control and Prevention, National Center for Injury Prevention and Control.

Forrest, G.M., Schwam, A.M., \& Cohen, E. (2002). Time of Care Required by Patients Discharged from a Rehabilitation Unit. American Journal of Physical Medicine \& Rehabilitation, 81, 57-62.

Huebner, R.A., Johnson, K., Miller Bennett, C., \& Schneck, C. (2003). Community Participation and Quality of Life Outcomes After Adult Traumatic Brain Injury. The American Journal of Occupational Therapy, 57, 177-185.

United States Department of Veterans Affairs. Traumatic Brain Injury and PTSD. Retrieved October 18, 2011 from https:// www.ptsd.va.gov/professional/treat/cooccurring/tbi_ptsd_vets.asp

Brain Injury Resources - C. E. Mckay \& M.E. Heffernan 